\title{
BMJ Open Sequential multiple assignment randomised trial of a brief contact intervention for suicide risk management among discharged psychiatric patients: an implementation study protocol
}

\author{
Huiming Liu, ${ }^{1}$ Guanjie Chen, ${ }^{1}$ Jinghua Li (D),${ }^{2}$ Chun Hao, ${ }^{2}$ Bin Zhang, ${ }^{1}$ \\ Yuanhan Bai, ${ }^{3}$ Liangchen Song, ${ }^{1}$ Chang Chen, ${ }^{1}$ Haiyan Xie, ${ }^{1}$ Tiebang Liu, ${ }^{1}$ \\ Eric D Caine, ${ }^{4}$ Fengsu Hou (10) ${ }^{1,2}$
}

To cite: Liu H, Chen G, Li J, et al. Sequential multiple assignment randomised trial of a brief contact intervention for suicide risk management among discharged psychiatric patients: an implementation study protocol. BMJ Open 2021;11:e054131. doi:10.1136/ bmjopen-2021-054131

- Prepublication history and additional supplemental material for this paper are available online. To view these files, please visit the journal online (http://dx.doi.org/10.1136/ bmjopen-2021-054131).

$\mathrm{HL}$ and GC are joint first authors.

Received 03 June 2021 Accepted 29 0ctober 2021

Check for updates

(c) Author(s) (or their employer(s)) 2021. Re-use permitted under CC BY-NC. No commercial re-use. See rights and permissions. Published by BMJ.

For numbered affiliations see end of article.

Correspondence to

Fengsu Hou;

houfengsu@gmail.com

\section{ABSTRACT}

Introduction The postdischarge suicide risk among psychiatric patients is significantly higher than it is among patients with other diseases and general population. The brief contact interventions (BCls) are recommended to decrease suicide risk in areas with limited mental health service resources like China. This study aims to develop a postdischarge suicide intervention strategy based on $\mathrm{BCls}$ and evaluate its implementability under the implementation outcome framework.

Methods and analysis This study will invite psychiatric patients and family members, clinical and community mental health service providers as the community team to develop a postdischarge suicide intervention strategy. The study will recruit 312 patients with psychotic symptoms and 312 patients with major depressive disorder discharged from Shenzhen Kangning Hospital (SKH) in a Sequential Multiple Assignment Randomised Trial. Participants will be initially randomised into two intervention groups to receive BCls monthly and weekly, and they will be rerandomised into three intervention groups to receive BCls monthly, biweekly and weekly at 3 months after discharge according to the change of their suicide risk. Follow-ups are scheduled at 1, 3, 6 and 12 months after discharge. With the intention-to-treat approach, generalised estimating equation and survival analysis will be applied. This study will also collect qualitative and quantitative information on implementation and service outcomes from the community team.

Ethics/dissemination This study has received ethical approval from the Ethics Committee Review Board of SKH. All participants will provide written informed consent prior to enrolment. The findings of the study will be disseminated through peer-reviewed scientific journals, conference presentations. A project report will be submitted to the National Natural Science Foundation of China as the concluding report of this funded project, and to the mental health authorities in the Shenzhen to refine and apply evidence-based and pragmatic interventions into health systems for postdischarge suicide prevention.
Strengths and limitations of this study

- To our knowledge, this is the first mix-methods study in China evaluating the implementation of an evidence-based intervention which reduces postdischarge suicide risk among psychiatric patients.

- A well-designed sequential multiple assignment randomised trial (SMART) is embedded to investigate the effectiveness of the brief contact intervention reducing postdischarge suicide risk among psychiatric patients.

- The community-based participatory research approach will be applied to develop the intervention strategy and to evaluate implementation outcomes.

- Although the sample size of SMART is well calculated and powered by previous studies, it is modest.

Trial registration number NCT04907669.

\section{INTRODUCTION}

Suicide is an acknowledged global public health concern. In China, the annual average suicide rate decreased from 23 per 100000 people between 1995 and 1999 to 6.75 per 100000 people between 2012 and $2015 .^{1-3}$ In 2017, as the fifth-leading cause of death, the reported suicide rate in China was 4.31 and 7.66 per 100000 people in urban and rural, respectively. ${ }^{4}$ In comparison, WHO reported the global rate was 10.5 per 100000 people in $2016 .^{5}$

Patients discharged from psychiatric settings carry substantially greater risk for suicide. The pooled rate of suicide among discharged psychiatric patients was 484 per 100000 person-years within 12 months worldwide, and it was 2950, 2060 and 1132 
per 100000 person-years within 1 week, 1 month and 3 months, respectively. ${ }^{6-15}$ We know of only one study involving persons of Chinese ethnicity, which found a rate of 1062 per 100000 people during the year following discharge in Hong Kong, where community mental health services (influence by programmes in the UK and in Australia) have been funded far more generously and, thus, been more resourceful in services than those in mainland China. ${ }^{8}$

There is no specific mental health policy in China with respect to psychiatric patients at risk of postdischarge suicide. For patients with severe mental disorders in China, which include schizophrenia, schizoaffective disorder, paranoid psychosis, bipolar disorder, psychotic disorders due to epilepsy or intellectual developmental disorder with psychotic disorders, they will receive follow-ups from community mental health workers after discharge according to the Code of Practice for the Management and Treatment of Severe Mental Disorders (2018 Edition).$^{16}$ In specific, the Code requires psychiatric facilities to report and register all patients with severe mental disorders in the Information Management System for Severe Mental Disorders, in which the patients will be rated from levels $0-5$ for the risk of violent behaviours. Registered patients will be rated as level 4 if conducted self-harm or attempted suicide, and the Code requires psychiatrists, family doctors, community mental health workers, mental health social workers, and the police to conduct joint follow-ups at least once every 2 weeks for patients at levels 3-5. However, the follow-ups focus on the risk of violent behaviours towards the public rather than postdischarge suicide.

For patients with other mental disorders, registrations in the system and joint follow-ups are not required. Psychiatrists may occasionally report individual patients with non-severe mental disorders who are at risk for suicide to the information system as appropriate; and once reported, community mental health workers must conduct follow-ups in accordance with the code focusing on suicide risk and related mental disorder symptoms. Other patients with suicide risk who are not reported will rely on active visit to outpatient clinics or contracting psychological crisis workers for postdischarge suicide interventions.

Brief contact interventions (BCIs) are evidence based and have been recommended to decrease postdischarge suicide risk in areas of limited mental health resources. ${ }^{1217-19}$ BCIs are a series of non-intrusive interventions at low cost aiming to develop long-term contact with discharged psychiatric patients by phone calls, caring letters, postcards, text messages, emergency green cards and crisis cards, etc. ${ }^{19-22}$ The key is to send messages to discharged patients (as well as their spouses and family members, relatives, friends and colleagues) at a predetermined frequency expressing greetings, encouragement, caring and support, and reminding them of psychological crisis assistance and mental health services. The proposed hypothesis of BCIs decreasing the postdischarge suicide risk is to increase patients' social connectedness and social support after discharge. ${ }^{23-26}$

WHO reported BCIs could decrease the postdischarge suicide risk among psychiatric patients effectively (OR $0.20,95 \%$ CI 0.09 to 0.42 ), and recommended integrating BCIs in the suicide intervention framework. ${ }^{12}$ In a randomised controlled trial (RCT) study, Motto reported the incidence of postdischarge suicide among intervention group was $8.48 \%(33 / 389)$ comparing with $14.10 \%(64 / 454)$ in control group ${ }^{27}$; however, in the followed 15-year cohort study, the significance of differences in postdischarge suicide incidence between groups wore off after 5 years. ${ }^{23}$ Similar RCT studies reported BCIs could decrease suicide ideation, the number of suicide attempts, the risk of self-harm and suicide death. ${ }^{17}$ 28-32 In China, studies usually applied BCIs as one component of comprehensive suicide intervention strategies, in which health education, consulting, assertive community treatment (ACT), and case management were also included, and reported effectiveness in reducing repeated attempted suicide, violent behaviours and improving compliance to treatments. ${ }^{33-39}$ However, few studies reported inconsistencies about the effectiveness of BCIs reducing postdischarge suicide ideation, attempts and deaths, which can be explained by different delivering frequencies (weekly, biweekly, monthly or quarterly), types of BCIs (calls, caring cards, emails or letters) and major outcomes (improvement of psychiatric symptoms, compliance to medication or postdischarge suicide). ${ }^{3740-44}$

In summary, most studies implemented BCIs monthly. Though few of them increased the delivering frequency from the first week to the first month after discharge, the frequency was reduced to monthly or bi-monthly, which could consequently be insufficient to maintain the effect on reducing postdischarge suicide risk in a long term. Hence, we hypothesise that BCIs with more intense delivering frequencies might work better for Chinese psychiatric patients than BCIs delivered monthly. Meanwhile, most of the content and the implementation strategy were predetermined by researchers rather than patients' needs and expectations. BCIs aim to reduce postdischarge suicide by increasing social connectedness and social support, but current studies did not measure the improvement of the two mediators or other confounding factors including socioeconomic factors, stigma, physical health and the use of mental health service, etc. Further, studies only evaluated the effectiveness and did not evaluate the feasibility and sustainability in daily work.

Hence, our specific aims include: (1) to develop an intervention strategy against postdischarge suicide risk for Chinese psychiatric patients based on BCIs; (2) to determine the best delivering frequency of BCIs for Chinese discharged psychiatric patients based on Sequential Multiple Assignment Randomised Trial (SMART) and (3) to evaluate its implementability under the Implementation Outcome Framework (IOF). 
Table 1 The definition of suicide behaviours in this study

\begin{tabular}{|c|c|}
\hline $\begin{array}{l}\text { Suicide } \\
\text { behaviours }\end{array}$ & Definition \\
\hline $\begin{array}{l}\text { Suicidal } \\
\text { ideation }\end{array}$ & $\begin{array}{l}\text { Having a clear intent to harm oneself without } \\
\text { a clear plan, nor taking any preparation or } \\
\text { actions. }\end{array}$ \\
\hline Suicidal plan & $\begin{array}{l}\text { Having a clear plan to harm oneself without } \\
\text { taking any preparation or actions. }\end{array}$ \\
\hline $\begin{array}{l}\text { Suicidal } \\
\text { preparation }\end{array}$ & $\begin{array}{l}\text { Taking any preparation to commit suicide } \\
\text { without taking actions to harm oneself. }\end{array}$ \\
\hline $\begin{array}{l}\text { Attempted } \\
\text { suicide }\end{array}$ & $\begin{array}{l}\text { Taking actions to commit suicide with a } \\
\text { certain intensity of wish to die, which did not } \\
\text { directly result in a fatal outcome. }\end{array}$ \\
\hline $\begin{array}{l}\text { Completed } \\
\text { suicide }\end{array}$ & $\begin{array}{l}\text { Taking actions to commit suicide with a } \\
\text { certain wish to die and directly resulting in } \\
\text { death. }\end{array}$ \\
\hline
\end{tabular}

\section{METHODS AND ANALYSIS}

This protocol has been written in accordance with the Standard Protocol Items for Randomised Trials statement and Consolidated Criteria for Reporting Qualitative Research checklist. ${ }^{45} 46$

In this study, we will adopt the definition of suicide behaviours in a behavioural continuum proposed by Professor Shuiyuan Xiao in the Chinese cultural context (table 1$).{ }^{47}$ We define suicide risk as the probability of an individual's death by suicide over a given time interval reflected by the intensity and frequency of suicide ideation, suicide plan, suicide preparation and suicide attempts. Suicide risk will be evaluated by the Beck Suicide Ideation Scale-Chinese Version (BSI-CV) and the suicidality module of the Mini-International Neuropsychiatric Interview (M.I.N.I-Suicidality).

\section{Prior study}

We conducted a prior study in Shenzhen Kangning Hospital (SKH) in early 2019. During 1 January to 31 March, there were 1349 discharged patients who aged 18 years and above, diagnosed with mental disorders based on the International Classification of Diseases 10th Revision (ICD-10), ${ }^{48}$ with ID, residence and source of income, and had been hospitalised for 3 days at least, and 689 of them were diagnosed with suicide risk at admission. Of 689 patients, 515 of them completed the survey in a 3-month follow-up. There were 20 attempted suicide cases and five completed suicide deaths, and the rate was 3883.5 (20/515) and $970.9(5 / 515)$ per 100000 people, respectively.

\section{Implementation science framework}

Evidence-based interventions and practices are poorly implemented, and it could take up to 17 years to adopt and integrate the interventions and practices into routine work by practitioners and policy-makers. ${ }^{49-51}$ To close the know-do gap and accelerate the implementation, implementation science aims to develop systematic methods and strategies to identify and address key

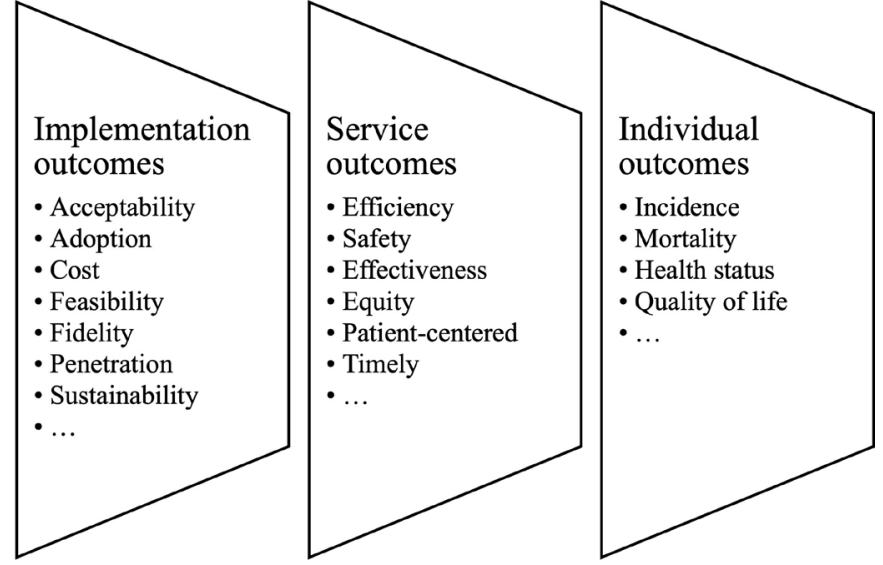

Figure 1 The implementation outcomes framework.

points that promote or impede the process. ${ }^{52}{ }^{53}$ We adopt the IOF that evaluates implementation strategies by implementation outcomes, service outcomes and client outcomes, including acceptability, sustainability, fidelity, efficiency, effectiveness, satisfaction and function etc (figure 1) ${ }^{5455}$ Based on IOF, we identify this study as a type-1 hybrid design implementation study that determines effectiveness and explores the context of routine implementation. ${ }^{56}$

\section{Study setting}

This study will be implemented in SKH, a public psychiatric hospital in Shenzhen City with over 1500 in-patient beds, 11590 person-time of in-patients, and 369000 person-time out-patient visits in 2020. Despite there are general hospitals providing psychiatric out-patient services in Shenzhen, SKH is the only medical facility providing in-patient psychiatric services. Shenzhen is with a population of 13.03 million residents, in which 8.48 million are internal migrants of varied sociodemographic features cross China. ${ }^{57}$ The reported life-time prevalence of any mental disorders (excluding dementia) in Shenzhen was $21.87 \%$, and the life-time prevalence of any mood disorders and any anxiety disorders was $9.62 \%$ and $14.45 \% .^{58}$ In comparison, the life-time prevalence of any mental disorders (excluding dementia), any mood disorders and any anxiety disorders was $16.60 \%, 7.40 \%$ and $7.60 \%$ in China, respectively. ${ }^{59}$

\section{Study design}

This is a mixed-methods study with two stages (figure 2). The first stage is to develop the intervention strategy by in-depth and focus group interviews; and the second stage is to implement the strategy and evaluate the implementation quantitatively by a randomised trial and qualitatively by focus group interviews. The anticipated start and end dates for the study are 1 September 2021 and 30 June 2023.

\section{The community-based participatory research}

We aim to recruit discharged psychiatric patients and their lay healthcare supporters (LHSs) who are usually family 


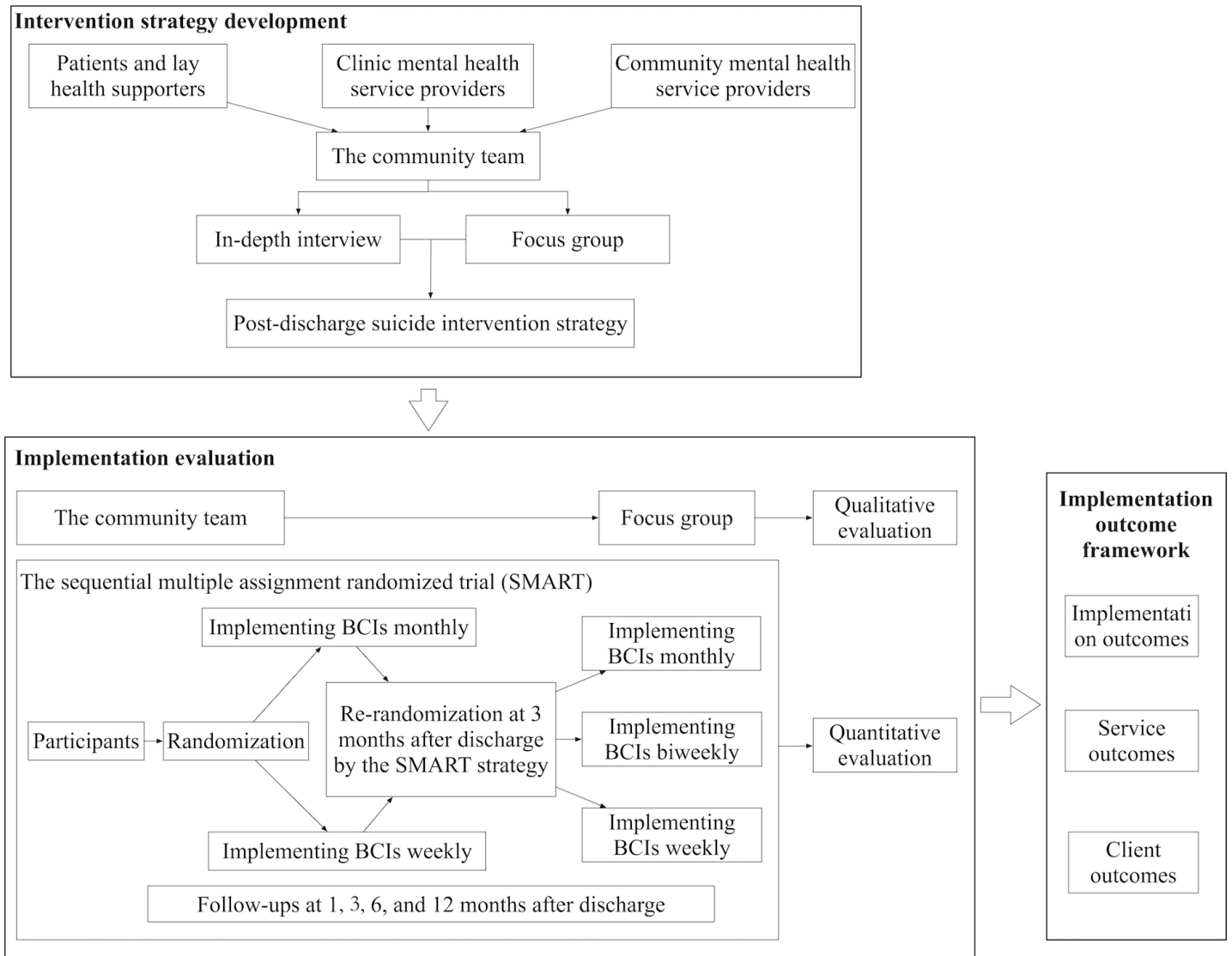

Figure 2 The summary of the study design. BCls, brief contact interventions.

members, psychiatrists and nurses, psychocrisis intervention team members, community mental health workers and mental health social workers as the community team that will provide a Chinese context under the communitybased participatory research (CBPR) framework. ${ }^{60-62}$ In specific, the framework would help this study:

- Explore the feasibility of implementing BCIs against suicide risk after discharge.

- Understand the needs for suicide risk management after discharge from related healthcare service providers and acceptors.

- Integrate suicide risk management experiences from the community.

- Discuss, develop and revise the intervention strategy with the community.

We categorise the community team into three subgroups, the patients-LHSs group and the clinic mental health service provider group (psychiatrists and nurses, and psychocrisis intervention team members) which will be recruited from SKH, and the community mental health service provider group (community mental health workers and mental health social workers) which will be recruited from eight community health centres in Shenzhen.

\section{Intervention development}

We will conduct three focus group interviews with each subgroup. To avoid bias in focus group interviews and to protect privacy related to personal experience in suicide and suicide intervention, we will also conduct ten to fifteen cases of in-depth interview in total with members from each subgroup. The themes include: (1) key points in suicide risk management after discharge, (2) how to develop BCIs content and deliver BCIs appropriately and feasibly to increase social connectedness and social support, and (3) how to improve compliance to treatment and increase subsequent visits after discharge. There will be scheduled meetings with the community to discuss and revise the intervention strategy before implementation.

\section{Implementation evaluation}

Based on IOF, we will conduct three focus group interviews in each subgroup to explore (1) patients' and LHSs' attitudes, acceptability and understanding of the strategy, (2) the clinic and community mental health service providers' willingness, feasibility and sustainability to implement the strategy and (3) the effectiveness, efficiency, equity, safety and timeliness of the strategy and whether it is patient-centred.

\section{The qualitative study sample}

Purposive sampling will be applied to recruit participants face-to-face for the community team. For each subgroup, there will be five to eight members. The inclusion criteria for the clinic and community mental health service provider groups are: (1) being 18 years and above and 


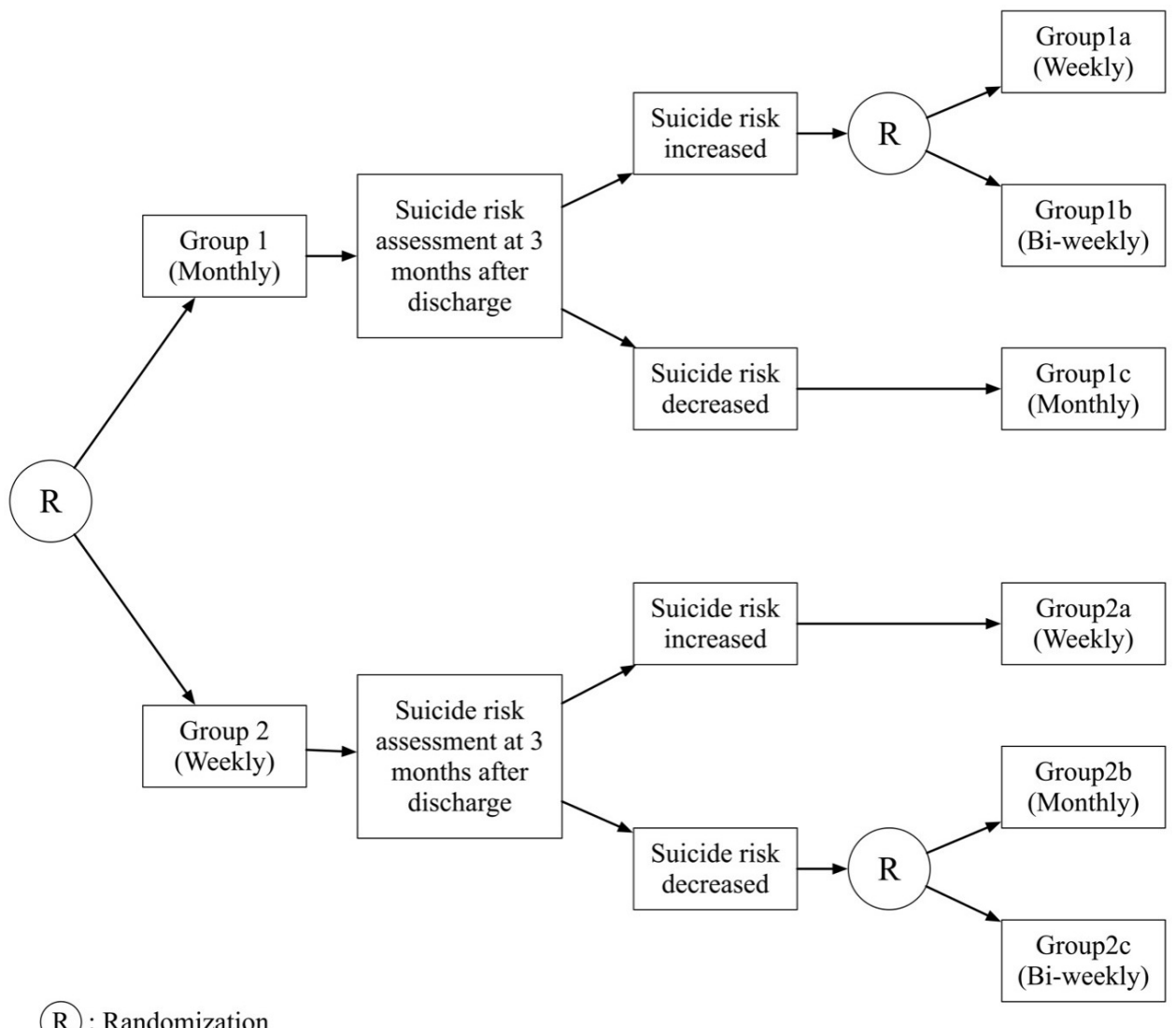

Figure 3 The smart design trial.

(2) having practiced in mental health service at least for 12 months. The inclusion criteria for the patients-LHSs group will be illustrated later. All participants will receive $¥ 100$ (about US\$15.42) to offset their efforts and cost of taking part.

\section{The qualitative data collection}

All coauthors from SKH have qualitative research experience and will conduct focus group and in-depth interviews in privacy-protected meeting rooms of SKH. There will be an interviewer, a recorder of field note, and an observer for interviews. The interviewer will introduce the aims of the study, the purpose of the interview and obtain written informed consent including audio recording consent before interviews begin (online supplemental file 1). The interview guide questions are showed in online supplemental file 2. Audiorecordings and field notes will be transcribed into text for analysis.

\section{The sequential multiple assignment randomised trial}

We will conduct the SMART to determine the best frequency to implement BCIs and investigate the patient outcomes in IOF. The SMART design reflects the idea of adaptive treatment strategies and dynamic treatment regimens that provide a sequence of decisions about the points at which to offer different interventions and a set of intervention options for each decision point. ${ }^{63-65}$ There will be two stages (figure 3).
Stage 1: After recruitment and baseline survey, participants will be randomised into group 1 and group 2 where BCIs will be implemented monthly and weekly, respectively. Because suicide risk is the highest in the first 3 months among discharged psychiatric patients, we set the check point at 3 months after discharge to assess participants' suicide risk in both groups.

Stage 2: At the check point, for participants in group 1 , if the suicide risk increased, they will be rerandomised into group 1a and group 1b where BCIs will be implemented weekly and biweekly, respectively; if the suicide risk decreased or did not change, they will remain receiving BCIs monthly as group 1c. For participants in group 2, if the suicide risk increased or did not change, they will remain receiving BCIs weekly as group 2a; if the suicide risk decreased, they will be rerandomised into group 2b and group 2c where BCIs will be implemented monthly and biweekly, respectively. After the rerandomisation, participants will continue to receive BCIs until 12 months after discharge, and the suicide risk will be evaluated at 1, 3, 6 and 12 months after discharge.

In this study, the magnitude of change in the total score of the BSI-CV or M.I.N.I-Suicidality that determines rerandomisation is 1 and above.

\section{The quantitative study sample}

We plan to implement the strategy in patients with psychotic symptoms and patients with major depressive 
disorder (MDD), as in representative of severe and nonsevere mental disorders.

The inclusion criteria for patients are: (1) being 18 years and above, (2) being diagnosed with psychotic symptoms or MDD based on the ICD-10, (3) having received inpatient care for 3 days or more, (4) living in Shenzhen and having no plan to leave Shenzhen in the following 12 months after discharge and (5) being able to read text messages, answer phone calls on mobile phones, use WeChat or any application on smart phones. WeChat is the most widely used app in China with about 11 billion active users in the first quarter of $2020{ }^{66}$ Considering participants' suicide risk, we will also recruit their LHSs to receive BCIs at the same frequency. The inclusion criteria are: (1) being 18 years and above, (2) without diagnosis of any mental disorder, (3) being the main lay healthcare supporter for the patient, (4) living in Shenzhen and having no plan to leave Shenzhen in the following 12 months after discharge and (5) being able to read text messages, answer phone calls on mobile phones, use WeChat, or any application on smart phones. All participants will receive ¥100 (about US\$15.42) to offset their efforts and cost of taking part.

Patients who are with cognitive impairment that prevents providing written informed consent due to either dementia or current psychosis episodes and who are with no ID, stable residence nor any source of income will be excluded. Particularly, patients discharged on families' or patients' demand against medical advice will be excluded.

\section{Sample size}

The sample size was calculated to estimate the primary effect between group 1 and group 2 in the trial. ${ }^{67}$ We set the rate of type I error $\alpha$ at 0.05 , the rate of type II error $\beta$ at 0.20 , the power $(1-\beta)$ at 0.80 , the moderate effect size $d$ at $0.35,{ }^{68}$ and the sample size is 130 for each group, 260 in total; considering dropout, we will increase the sample size by $20 \%$, and the final sample size is 312 participants. We will conduct two SMART trials in patients with psychotic symptoms and MDD separately, and the sample size for each trial is 312 (624 patients in total). We aim to recruit participants from 1 January 2022 until the sample size is reached.

\section{Randomisation and mask}

After recruitment and the baseline survey, we will assign participants into group 1 and group 2 by block randomisation in $\mathrm{R}$ program. ${ }^{69}$ At the check point in the SMART trial, we will reassign participants into group 1a, group $1 b$, group 1c, group 2a, group $2 b$ and group $2 c$ based on their suicide risk by simple randomisation in $\mathrm{R}$ program. The allocation ratio in randomisation will be $1: 1$. The randomisation will be performed by a statistician in the research team. Patients, LHSs, nurses who perform recruitment and baseline survey, the statistician who performs randomisation, and investigators who perform follow-ups will be blinded to the assignment.

\section{Brief contact intervention}

The BCI in this study is a series of structured messages, and it will primarily be delivered through pushing feeds on WeChat due to its popularity in China, and an iOS/ Android application will also be applied to deliver the intervention. If participants did not use smartphones, messages will be delivered by mobile text messages or by phone calls. Though the content of messages is yet to be determined by the CBPR study, we expect to structure messages into six components including introduction, greetings for previous complaints, mental health promotion, encouragement and coping strategies, remind of treatment and subsequent visit, and crisis intervention resource. Noted, the same messages will also be sent to LHSs. Figure 4 shows an example of the BCI delivered through WeChat.

\section{Quantitative data collection}

To evaluate postdischarge suicide risk more cautiously and to provide crisis intervention in time, we will conduct face-to-face interview to collect information. After research assistants introduce the study and obtain written informed consent, trained nurses in SKH will recruit participants and perform baseline survey before discharge. As mentioned, we encourage subsequent visits to SKH out-patient clinics in BCIs, and research assistants will contact participants to schedule outpatient visits and complete follow-up surveys during the visits at 1, 3, 6 and 12 months after discharge. If participants refused follow-ups in out-patient settings, we would schedule home visits to complete the survey by research assistants and community mental health workers. If patients did not respond, research assistants will contact their LHSs to obtain participants' recent updates and help them schedule outpatient visits for patients if necessary. Drop-out is defined as (1) participants or their LHSs request to quit the study and stop receiving any brief contact messages; (2) participants or their LHSs refuse follow-up surveys either at outpatient clinics or at home and (3) participants pass away by accidents or other health problems except suicide. Particularly, at each time point of follow-ups, we will contact patients and LHSs up to three times. If neither of them responded, they would be treated as dropout.

\section{Study outcomes and measurements}

The study outcomes are based on the implementation outcomes framework.

\section{Implementation outcomes}

Acceptability and adoption will be evaluated by the community's attitudes generating from qualitative interviews. The adoption rate will be measured by the number of participants who subscribe to follow the study's WeChat Platform or the iOS/Android smartphone application divided by the number of participants who remain as followers at the end of the study.

Feasibility will be evaluated by mental health service providers' attitudes generated from qualitative interviews. 


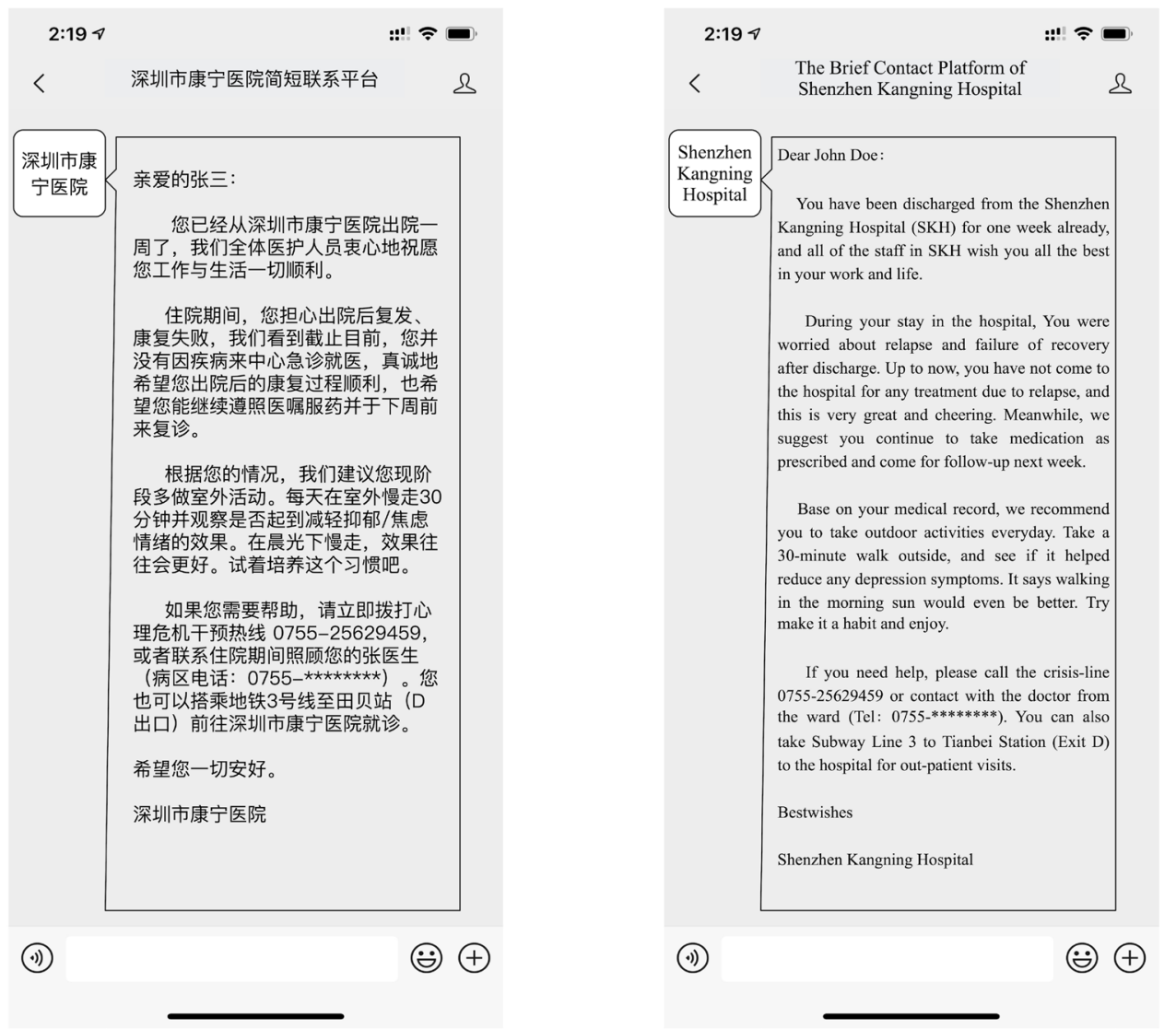

Figure 4 An example of the brief contact intervention delivered through WeChat.

Cost will be measured by the total cost of implementing the SMART trial, which will be recorded to assess the economic benefits of the intervention during the study.

Fidelity will be measured by a staged checklist that evaluates the degree to which the study is implemented as described in the protocol, the quality, and the competence of the study.

Sustainability will be evaluated by mental health service providers' attitudes generated from qualitative interviews.

\section{Service outcomes}

Efficiency will be measured by the number of daily brief contacts delivered to participants through WeChat, the application, text messages and phone calls during implementation.

Safety that whether implementing BCIs would be any potential harm/danger to patients will be evaluated by the community's attitudes generated from qualitative interviews.

Effectiveness will be measured by the comparison of the trajectories of suicide ideation and suicidality from baseline to 3 and 12 months after discharge between group 1 and group 2, respectively.

Equity will be evaluated by the community in focus group interviews that how the intervention strategy considers and address the disparities in social groups.

Patient-centredness be evaluated by the community in focus group interviews that how well the intervention strategy considers and meets the needs and demands of patients, and whether the strategy fully considers participants' feelings.

Timeliness will be measured by the time that the research team cost to respond to participants' feedbacks and requests for crisis intervention.

\section{Client outcomes}

The trajectories of suicide risk (suicide ideation and suicidality) from baseline to 3-month and 12 month postdischarge are the primary outcomes. The trajectories of suicide risk from 3-month to 12-month postdischarge are the secondary outcomes. The trajectories of social connectedness and social support from baseline to 3-month and 12-month postdischarge are the secondary outcomes.

Suicide ideation will be measured by the BSI-CV, which has been translated and modified in the Chinese context, and it has been validated and widely applied in China. ${ }^{70-75}$ The BSI-CV includes 19 items evaluating specific attitudes, ideations, behaviour and plans to commit suicide during the past week, and each item scores from 0 to 2 with a total score ranging from 0 to 38 , and a higher score indicates higher level of suicide risk.

Suicidality will be measured by the suicidality module of the M.I.N.I.-Suicidality, which has been validated in China, to assess suicide risk for inpatients and outpatients, we will also evaluate participants' suicidality by this scale. ${ }^{76-78}$ In the 6-item scale, dichotomous items ('No' or 'Yes') evaluate wish to be dead, self-hurt, suicide ideation, 
plan, current and ever attempts during the past month, and 'yes' to each item is assigned to score 1, 2, 6, 10, 10 and 4, respectively, with a higher total score indicating higher level of suicide risk.

Social connectedness will be measured by the Social Connectedness Scale (SCS) to evaluate participants' social connected ness after discharge, which has been validated in China. ${ }^{790}$ The SCS is a 20 -item scale, and each item is on a 6-Likert continuum (from 'Strongly disagree' to 'Strongly agree') scoring from 1 to $6{ }^{80} \mathrm{~A}$ higher total score indicates a higher level of social connectedness.

Social support will be measured by the 23-item Duke Social Support Index (DSSI) to evaluate participants' social support after discharge. ${ }^{81}$ The Chinese version of DSSI has been validated and applied in China. ${ }^{82-85}$ The DSSI investigates social support by social interaction, perceived social support and instrumental social support. Every answer has been assigned a score, and the total reflects the sum of the items ranging from 11 to 45. A higher total score indicates a higher level of social support.

\section{Covariates}

We will develop a questionnaire to collect information about covariates, and the questionnaire will be validated in pilot.

Demographic information will be collected at baseline by self-made questionnaire including age, marital status, occupation, income, Hukou (household residence registration) and residence time in Shenzhen.

Times of rehospitalisation for mental disorders will be measured by responses to the question 'How many times have you been hospitalised for mental disorders?' in follow-ups.

The usage of crisis intervention will be measured by the responses to the question 'How many times have you called the research team or the Crisis Intervention Hotline for help after discharged from hospital?' in follow-ups.

Perceived stigma will be evaluated by the Chinese version of Link Perceived Devaluation-Discrimination Scale. ${ }^{86}{ }^{87}$ The scale contains 12 items assessing the extent to which a person believes that other people will devalue or discriminate against someone with a mental illness. Each item is on a 4-Likert continuum (from 'Strongly disagree' to 'Strongly agree') scoring from 1 to 4 . A higher total score indicates a higher level of perceived stigma. The trajectories of patients' perceived stigma from baseline to 3-month and 12-month after discharge will be analysed.

Self-efficacy will be evaluated by the Chinese version of the General Self-Efficacy Scale. ${ }^{88}$ The scale contains 10 items, and each item is on a 4-Likert continuum (from 'Not at all true' to 'Exactly true') scoring from 1 to 4 . A higher total score indicates a higher level of self-efficacy. The total score's trajectory from baseline to 3 months after discharge will be recorded and compared. The trajectories of patients' self-efficacy from baseline to 3-month and 12-month after discharge will be analysed.
Compliance to treatment will be evaluated by a 4 -item self-administered questionnaire. The questionnaire inquires whether the patients take medications under the instruction on prescriptions. Each item is on a 4-Likert continuum (from 'Not following the instruction' to 'Exactly following the instruction') scoring from 1 to 4 . A higher total score indicates a higher level of compliance to treatment. The change of patients' compliance from baseline to 3-month and 12-month after discharge will be analysed.

\section{Statistical analyses}

We will perform the in analyses. Demographic and baseline information between participants in group 1 and group 2, as well as between participants with psychotic symptoms and MDD, will be presented in the form of mean (SD), the $95 \%$ CIs for continuous variables, and percentages for categorical variables.

We will use independent t-test (for continuous variables) and $\chi^{2}$ test (categorical variables) to compare the differences between groups. We will use generalised estimating equation (GEE) to explore the time-trends/ trajectories of repeated measured outcomes and adjust for potential confounding variables.

We will use survival analyses to compare the effect of BCIs reducing postdischarge suicide risk at endpoint between participants in group 1 and group 2, as well as between patients with psychotic symptoms and MDD. The model will take mediating factors into account. We will run pairwise comparisons between reassigned groups by GEE ((group 1a+group 1c] vs (Group 1b+group 1c) vs (group 2a+group 2b) vs (group 2a+group 2c)). And we will use path analysis to validate the hypothesis that BCIs could decrease postdischarge suicide risk by increasing social connectedness and social support. Further, we plan to use the Bootstrap percentile method to calculate the Average Cost-Effectiveness Ratio that reflects the cost of reducing one unit of postdischarge suicide risk (one unit of score in BSI-CV and M.I.N.I.-Suicidality).

Multiple imputation will be used to account for the missing values, assuming they are missing at random. We set statistical significance at 0.05 and all analyses will be two sided. All data analyses will be performed in the R program. ${ }^{68}$

\section{Qualitative analyses}

We will code the qualitative data into the categorical and numerical data with a three-step procedure, and then apply content analysis method to analyse data in $\mathrm{R}$ program. ${ }^{89} 90$

Open coding: Four coders will independently code the qualitative data into categorical and numerical codes, and share their codes. If the codes were different over the same response, there would be a discussion until reaching consensus.

Axial coding: During analysis, the authors will associate codes to each other, and reconceptualised categories and subcategories to fully elaborate codes.

Selective coding: The authors will compare different categories of codes and examined the associations to 
identify a core category that could represent the key themes to research questions and related to other categories. The selective coding is at a higher level compared with axial coding, and the core category could be a new category created during analysis.

Lastly, we will enter the categorical and numerical data into a database for content analysis and generated the qualitative results.

\section{Data monitoring and quality assurance}

The study will receive overall supervision from the Department of Research and Education Management in SKH, who will quarterly monitor the progress and review the quality and completeness of data. All data will be stored at encrypted password-protected storage devices owned by SKH, and only the research team members have the access to view, manage and analyse. Nurses who recruit participants and conduct baseline survey and research assistants will be responsible for identifying and recruiting participants, obtaining informed written consent and performing double data entry. A formal data monitoring committee will not be considered for the conduct of this study as this is a low-risk intervention; however, the study will be annually reviewed by the Ethics Committee Review Board in SKH.

\section{Ethics and dissemination}

The study protocol (10 May 2021, V.1.1) has received approval from the Ethics Committee Review Board of SKH, and any violations of the study protocol will be recorded and reported to the board.

The findings of the study will be disseminated through peer-reviewed scientific journals and conference presentations. A conclusion report will be submitted to the National Natural Science Foundation of China and the Shenzhen Municipal Health Commission.

\section{Patient and public partnership involvement}

In this study, we will apply the CBPR principles which allow patients, family members and mental health service providers to participate in developing and evaluating the intervention strategy against postdischarge suicide.

\section{DISCUSSION}

To our knowledge, this study is the first implementation study in China to recruit a sizeable number of in-hospitalised patients with psychotic symptoms and MDD in a communitybased participatory setting and a continuum of mental healthcare aiming to decrease postdischarge suicide risk. We believe the results may provide implementational evidence for stakeholders in China on reducing postdischarge suicide risk for psychiatric patients in resource-limited areas.

Interventions that reduce postdischarge suicide risk among psychiatric patients usually apply BCIs, psychological therapies (ie, behaviour therapy, cognitive therapy and behaviour cognitive therapy), medication treatment, case management and ACT. ${ }^{189192}$ Though interventions like case management and ACT are effective to prevent postdischarge suicide, they are more viable and practical in countries/regions with adequate mental health and social resources, and it is not suitable for widespread implementation in China, where there are about 2.20 psychiatric professionals per 100000 persons including psychiatrists and community mental health workers. ${ }^{93}{ }^{94}$ In Shenzhen, there are 2.50 psychiatric professionals per 100000 persons, which is lower than that in Canada (14.68), the USA (10.54) and Japan (11.87). ${ }^{93} 95$ Hence, it is necessary to explore implementation effectiveness of low-cost interventions like BCIs in China.

Short length of stay, side effects of medication treatments, low treatment adherence, history of suicide attempts and hospitalisation and discharge experiences were associated with increased suicide risk among discharged psychiatric patients. ${ }^{96}$ Meanwhile, studies also report the loneliness, feelings of lost and uncertainty would increase postdischarge suicide risk: (1) patients are aware of suicide risk, but they do not know how to manage it and neither how nor whom to ask for help; (2) without doctor's or nurse's orders/advice, patients may lose daily goals and don't know what to do after discharge; (3) patients may actively avoid contact with others and feel lonely even if others take the initiative to care; (4) patients may experience self-blame and self-guilt and (5) patients may experience frustrations in recovery. ${ }^{23-25}$ These studies not only provide a context that explain the high postdischarge suicide risk among psychiatric patients, but also indicate the importance of social connectedness and social support that BCIs could deliver to decrease the risk.

This study has several strengths. First, it addresses the continuum of mental healthcare from clinic to postdischarge settings and emphasises on social connectedness and social support. Second, the study focuses on implementation outcomes. We will not only focus on the decrease of postdischarge suicide risk but also the acceptability, adoption, fidelity, efficiency, safety, equity and patient-centredness, etc. Third, the study will apply the CBPR framework to develop a culturally tailored and locally contextual intervention strategy, which will fully consider benefits of all stakeholders (patients and family members, clinic and community mental health service providers) in postdischarge suicide risk management. Fourth, we will apply the SMART design to explore the effect of BCIs on decreasing postdischarge suicide risk and to determine the best frequency to deliver BCIs. The SMART design could improve validity by allowing simultaneous evaluation of the results of different interventions or combinations of interventions, reduce dropouts by reassigning participants who are not sensitive to the initial intervention or do not have the desired outcome to another intervention, examine what intervention participants have received and when, and promise all participants receive interventions. ${ }^{63-65}$

Although this study may hold promise for better implementation, service and client outcomes, there are potential limitations. Though we will have a sample size with the power to detect outcomes, we will only recruit patients with psychotic symptoms and MDD who cannot be the represent all patients discharged from psychiatric settings, while the setting of the study is in Shenzhen that may not represent 
the entire China. As a type-1 hybrid design implementation study, there are outcomes predominantly being evaluated by qualitative interviews, including feasibility, acceptability and sustainability, which may not fully represent the implementation in practice. Thus, the generalisability of our findings will be limited.

\section{Author affiliations}

${ }^{1}$ Department of Public Health, Shenzhen Kangning Hospital, Shenzhen, Guangdong, China

${ }^{2}$ Sun Yat-sen Global Health Institute, Sun Yat-Sen University School of Public Health, Guangzhou, Guangdong, China

${ }^{3}$ Department of Bipolar Disorders, Shenzhen Kangning Hospital, Shenzhen, Guangdong, China

${ }^{4}$ Department of Psychiatry, University of Rochester Medical Center, Rochester, New York, USA

Acknowledgements The authors thank our team members from Shenzhen Kangning Hospital and Sun Yat-sen University for their contribution to this study.

Contributors All authors contributed to the conceptualisation and the design of this study. FH obtained the funding and contributed to the theoretical framework of the study. FH and $\mathrm{HL}$ conceived the prototype of the intervention, the study design, and the creation of the team. $\mathrm{HL}$ and GC drafted the initial manuscript together. $\mathrm{JL}$ and $\mathrm{CH}$ provided the sampling, randomisation and analytical strategy. $\mathrm{BZ}$ and $\mathrm{YB}$ conceived the content of the intervention and provided crisis intervention service in the study. LS, CC and HX contributed to the implementation of the study. TL and EDC steered the direction of the study and contributed significantly to the revision of the manuscript. All authors read and revised the initial manuscript and approved the final version.

Funding This study is funded by the National Natural Science Foundation of China (Grant No. 7004140).

Competing interests None declared.

Patient consent for publication Not applicable.

Provenance and peer review Not commissioned; externally peer reviewed.

Supplemental material This content has been supplied by the author(s). It has not been vetted by BMJ Publishing Group Limited (BMJ) and may not have been peer-reviewed. Any opinions or recommendations discussed are solely those of the author(s) and are not endorsed by BMJ. BMJ disclaims all liability and responsibility arising from any reliance placed on the content. Where the content includes any translated material, BMJ does not warrant the accuracy and reliability of the translations (including but not limited to local regulations, clinical guidelines, terminology, drug names and drug dosages), and is not responsible for any error and/or omissions arising from translation and adaptation or otherwise.

Open access This is an open access article distributed in accordance with the Creative Commons Attribution Non Commercial (CC BY-NC 4.0) license, which permits others to distribute, remix, adapt, build upon this work non-commercially, and license their derivative works on different terms, provided the original work is properly cited, appropriate credit is given, any changes made indicated, and the use is non-commercial. See: http://creativecommons.org/licenses/by-nc/4.0/.

\section{ORCID iDs}

Jinghua Li http://orcid.org/0000-0002-9571-7359

Fengsu Hou http://orcid.org/0000-0001-5869-4365

\section{REFERENCES}

1 Phillips MR, Li X, Zhang Y. Suicide rates in China, 1995-99. The Lancet 2002;359:835-40.

2 Liu Z, Huang Y, Ma C. Suicide rate trends in China from 2002 to 2015. Chinese Mental Health Journal 2017;31:756-67.

3 Jiang $\mathrm{H}$, Niu L, Hahne $\mathrm{J}$, et al. Changing of suicide rates in China, 2002-2015. J Affect Disord 2018;240:165-70.

4 National Health Commission of the People's Republic of China. Yearbook of Health in the People's Republic of China. Beijing: Peking Union Medical College Press, 2018.

5 World Health Organization. Global health Observatory (GHO) data 2017. Available: https://www.who.int/gho/mental_health/suicide rates/en/ [Accessed 28 Jun 2020].
6 Goldacre M, Seagroatt V, Hawton K. Suicide after discharge from psychiatric inpatient care. The Lancet 1993;342:283-6.

7 Geddes JR, Juszczak E. Period trends in rate of suicide in first 28 days after discharge from psychiatric hospital in Scotland, 1968-92. BMJ 1995;311:357-60.

8 Appleby L, Shaw J, Amos T, et al. Suicide within 12 months of contact with mental health services: national clinical survey. $B M J$ 1999;318:1235-9.

9 Meehan J, Kapur N, Hunt IM, et al. Suicide in mental health in-patients and within 3 months of discharge. British Journal of Psychiatry 2006;188:129-34.

10 Hunt IM, Kapur N, Webb R, et al. Suicide in recently discharged psychiatric patients: a case-control study. Psychol Med 2009;39:443-9.

11 Bickley H, Hunt IM, Windfuhr K, et al. Suicide within two weeks of discharge from psychiatric inpatient care: a case-control study. Psychiatr Serv 2013;64:653-9.

12 World Health Organization. Preventing suicide: a global imperative. World Health Organization, 2014. https://www.who.int/mental_health/ suicide-prevention/world report_2014/en/

13 Chung DT, Ryan CJ, Hadzi-Pavlovic D, et al. Suicide rates after discharge from psychiatric facilities: a systematic review and metaanalysis. JAMA Psychiatry 2017;74:694.

14 Haglund A, Lysell $\mathrm{H}$, Larsson $\mathrm{H}$, et al. Suicide immediately after discharge from psychiatric inpatient care: a cohort study of nearly 2.9 million discharges. J Clin Psychiatry 2019;80. doi:10.4088/ JCP.18m12172. [Epub ahead of print: 1202 2019].

15 Chung D, Hadzi-Pavlovic D, Wang M, et al. Meta-Analysis of suicide rates in the first week and the first month after psychiatric hospitalisation. BMJ Open 2019;9:e023883.

16 National health commission of People's Republic of China. The Code of Practice for the Management and Treatment of Severe Mental Disorders (2018 Edition): National health commission of People's Republic of China 2018. Available: http://www.nhc.gov.cn/jkj/s7932/ 201806/90d5fe3b7f48453db9b9beb85dfdc8a8.shtml [Accessed 23 Sep 2019].

17 Fleischmann A, Bertolote JM, Wasserman D, et al. Effectiveness of brief intervention and contact for suicide attempters: a randomized controlled trial in five countries. Bull World Health Organ 2008;86:703-9.

18 Zalsman G, Hawton K, Wasserman D, et al. Suicide prevention strategies revisited: 10-year systematic review. Lancet Psychiatry 2016;3:646-59.

19 Riblet NBV, Shiner B, Young-Xu Y, et al. Strategies to prevent death by suicide: meta-analysis of randomised controlled trials. $\mathrm{Br} \mathrm{J}$ Psychiatry 2017:210:396-402.

20 Luxton DD, June JD, Comtois KA. Can postdischarge follow-up contacts prevent suicide and suicidal behavior? A review of the evidence. Crisis 2013;34:32-41.

21 Falcone G, Nardella A, Lamis DA, et al. Taking care of suicidal patients with new technologies and reaching-out means in the postdischarge period. World J Psychiatry 2017;7:163-76.

22 Messiah A, Notredame C-E, Demarty A-L, et al. Combining green cards, telephone calls and postcards into an intervention algorithm to reduce suicide reattempt (AlgoS): P-hoc analyses of an Inconclusive randomized controlled trial. PLoS One 2019:14:e0210778.

23 Motto JA, Bostrom AG. A randomized controlled trial of postcrisis suicide prevention. Psychiatr Serv 2001;52:828-33.

24 Cutcliffe JR, Links PS, Harder HG, et al. Understanding the risks of recent discharge: the phenomenological lived experiences-"existential angst at the prospect of discharge". Crisis 2012;33:21-9.

25 Riblet N, Shiner B, Scott R, et al. Exploring psychiatric inpatients beliefs about the role of post-discharge follow-up care in suicide prevention. Mil Med 2019;184:e91-100.

26 Hare-Duke L, Dening T, de Oliveira D, et al. Conceptual framework for social connectedness in mental disorders: systematic review and narrative synthesis. J Affect Disord 2019;245:188-99.

27 Motto JA. Suicide prevention for high-risk persons who refuse treatment. Suicide Life Threat Behav 1976;6:223-30.

28 Carter GL, Clover K, Whyte IM, et al. Postcards from the edge project: randomised controlled trial of an intervention using postcards to reduce repetition of hospital treated deliberate self poisoning. BMJ 2005;331:805.

29 Carter GL, Clover K, Whyte IM, et al. Postcards from the edge: 24-month outcomes of a randomised controlled trial for hospitaltreated self-poisoning. Br J Psychiatry 2007;191:548-53.

30 Cebrià Al, Parra I, Pàmias M, mias M P, et al. Effectiveness of a telephone management programme for patients discharged from an emergency department after a suicide attempt: controlled study in a Spanish population. J Affect Disord 2013;147:269-76. 
31 Hassanian-Moghaddam H, Sarjami S, Kolahi A-A, et al. Postcards in Persia: a twelve to twenty-four month follow-up of a randomized controlled trial for hospital-treated deliberate self-poisoning. Arch Suicide Res 2017:21:138-54.

32 Comtois KA, Kerbrat AH, DeCou CR, et al. Effect of augmenting standard care for military personnel with brief caring text messages for suicide prevention: a randomized clinical trial. JAMA Psychiatry 2019;76:474-83.

33 Zhang S, Zhou G. Intervention of violent and suicidal behaviour of mental patients in the community. Journal of Clinical Psychological Medicine 1999;9:77-8.

34 Fu Y, Shen J, Dang W. Effects of psychological intervention on the young people of attempted suicide. Chinese Mental Health Journal 2007;21:571-4.

$35 \mathrm{Bi} \mathrm{B}$. Intervention for emergency department patients who attempt suicide in ShenYang General Hospital: an 1-year follow-up study. China Medical University, 2010.

36 Xu D, Zhang X, Li X. Effectiveness of 18-month psychosocial intervention for suicide attempters. Chinese Mental Health Journal 2012;26:24-9.

37 Chen W-J, Ho C-K, Shyu S-S, et al. Employing crisis postcards with case management in Kaohsiung, Taiwan: 6-month outcomes of a randomised controlled trial for suicide attempters. BMC Psychiatry 2013;13:191.

38 Zhao W, Peng M, Zhao Y. The mechanism of assertive community treatment effects on the suicidality in schizophrenia patients. Chinese Journal of Clinical Psychology 2018;26:620-3.

39 Yuan Q, Qian M, Tao L. The effect of individualized information support on suicidal attitude in patients with depressive disorder. Chinese Primary Health Care 2019;33:45-8.

40 Cotgrove A, Zirinsky L, Black D, et al. Secondary prevention of attempted suicide in adolescence. J Adolesc 1995;18:569-77.

41 Evans J, Evans M, Morgan HG, et al. Crisis card following self-harm: 12-month follow-up of a randomised controlled trial. Br J Psychiatry 2005;187:186-7.

42 Vaiva G, Walter M, Al Arab AS, et al. ALGOS: the development of a randomized controlled trial testing a case management algorithm designed to reduce suicide risk among suicide attempters. BMC Psychiatry 2011;11:11(1):1.

43 Reger MA, Luxton DD, Tucker RP, et al. Implementation methods for the caring contacts suicide prevention intervention. Prof Psychol 2017;48:369-77.

44 Yuan Y, Wang G, Shen L. The effect of suicide risk for implementing hospital-community-family integrated care in depression patients. Chinese Nursing Research 2016;30:4174-6.

45 Tetzlaff JM, Moher D, Chan A-W. Developing a guideline for clinical trial protocol content: Delphi consensus survey. Trials 2012;13:176

46 Tong A, Sainsbury P, Craig J. Consolidated criteria for reporting qualitative research (COREQ): a 32-item checklist for interviews and focus groups. Int J Qual Health Care 2007;19:349-57.

47 Xiao S, Zhou L, Xu H. Suicide prevention and crisis intervention -the concept and certification of suicide. Journal of Clinical Psychiatry 2005;15:298-9.

48 World Health Organization. The ICD-10 classification of mental and behavioural disorders: diagnostic criteria for research. Geneva: World Health Organization, 1993.

49 Balas EA, Boren SA. Managing clinical knowledge for health care improvement. Yearb Med Inform 2000;09:65-70.

50 Grant J, Green L, Mason B. Basic research and health: a reassessment of the scientific basis for the support of biomedical science. Res Eval 2003;12:217-24.

51 Morris ZS, Wooding S, Grant J. The answer is 17 years, what is the question: understanding time lags in translational research. $J R$ Soc Med 2011;104:510-20.

52 Eccles MP, Mittman BS. Welcome to implementation science. Implementation Science 2006;1.

53 Bauer MS, Damschroder L, Hagedorn $\mathrm{H}$, et al. An introduction to implementation science for the non-specialist. BMC Psychol 2015;3:32.

54 Proctor EK, Landsverk J, Aarons G, et al. Implementation research in mental health services: an emerging science with conceptual, methodological, and training challenges. Adm Policy Ment Health 2009;36:24-34.

55 Proctor E, Silmere H, Raghavan R, et al. Outcomes for implementation research: conceptual distinctions, measurement challenges, and research agenda. Adm Policy Ment Health 2011;38:65-76.

56 Curran GM, Bauer M, Mittman B, et al. Effectiveness-implementation hybrid designs: combining elements of clinical effectiveness and implementation research to enhance public health impact. Med Care 2012;50:217-26.
57 Statistics Bureau of Shenzhen Municipality. Shenzhen statistical Yearbook 2019. Beijing: China Satatistics Press Co, 2019.

$58 \mathrm{Hu}$ J-Z, Hu C-Y, Duan W-D, et al. [Survey on mental disorders among registered residents and non-registered residents in Shenzhen]. Zhonghua Liu Xing Bing Xue Za Zhi 2009;30:543-8.

59 Huang $\mathrm{Y}$, Wang $\mathrm{Y}$, Wang $\mathrm{H}$, et al. Prevalence of mental disorders in China: a cross-sectional epidemiological study. Lancet Psychiatry 2019;6:211-24.

60 Israel BA, Schulz AJ, Parker EA, et al. Review of community-based research: assessing partnership approaches to improve public health. Annu Rev Public Health 1998;19:173-202.

61 Israel BA, Schulz AJ, Parker EA, et al. Community-Based participatory research: policy recommendations for promoting a partnership approach in health research. Educ Health 2001;14:182-97.

62 Smikowski J, Dewane S, Johnson ME, et al. Community-Based participatory research for improved mental health. Ethics Behav 2009;19:461-78.

63 Murphy SA. An experimental design for the development of adaptive treatment strategies. Stat Med 2005;24:1455-81.

64 Lei H, Nahum-Shani I, Lynch K, et al. A "SMART" design for building individualized treatment sequences. Annu Rev Clin Psychol 2012;8:21-48.

65 Wallace MP, Moodie EEM, Stephens DA. Smart thinking: a review of recent developments in sequential multiple assignment randomized trials. Current Epidemiology Reports 2016;3:225-32.

66 Tencent Holdings Ltd. The 2020 first quarter results 2020. Available: https://cdc-tencent-com-1258344706.image.myqcloud.com/ uploads/2020/05/18/fc1afc176e4604f3a05602a467b259ad.pdf [Accessed 28 Jul 2020].

67 Crivello A, Levy J, Murphy S. Statistical methodology for a smart design in the development of adaptive treatment strategies: University Park, PA: the Pennsylvania state University, the methodology center, 2007.

68 Cohen J. Statistical power analysis for the behavioural sciences. Hillsdale, NJ: Laurence Erlbaum Associates: Inc, 1988.

69 R: A language and environment for statistical computing [program] 2013.

70 Li X, Phillips MR, Zhang Y. Reliability and validity of the Chinese version of Beck Scate for suicide ideation (BSI-CV) among university students. Chinese Mental Health Journal 2011;25:862-6.

$71 \mathrm{He} \mathrm{H}$, Yang Y. Path analysis on the influencing factors of suicide ideation among college students in Beijing. Chinese Journal of School Health 2015;36:80-3.

72 Ai M, Chen JM, Wang MJ. The impulsiveness and aggression among suicide attempters in college students. Chinese Journal of Nervous and Mental Diseases 2011;37:650-5.

73 Zhou ZJ, xi Y, Liu TB. Influencing factors to suicide behaviors among adolescent students of four schools in Shenzhen. Chinese Journal of School Health 2015;36:1330-3.

74 Beck AT, Steer RA, Ranieri WF. Scale for suicide ideation: psychometric properties of a self-report version. J Clin Psychol 1988;44:499-505.

$75 \mathrm{Li}$ X, Phillips MR, Tong YS. Reliability and validity of the Chinese version of Beck suicide ideation scale (BSI-CV) in adult community residents. Chinese Mental Health Journal 2010;24:250-5.

76 Sheehan DV, Lecrubier Y, Sheehan KH, et al. The Mini-International neuropsychiatric interview (M.I.N.I.): the development and validation of a structured diagnostic psychiatric interview for DSM-IV and ICD10. J Clin Psychiatry 1998;59 Suppl 20:22-33.

77 Si T, Shu L, Dang W. Evaluation of the reliability and validity of Chinese version of the Mini.International neuropsychiatric interview in patients with mental disorders. Chinese Mental Health Journal 2009;23:493-7.

78 Lecrubier Y, Sheehan DV, Weiller E, et al. The mini international neuropsychiatric interview (mini). A short diagnostic structured interview: reliability and validity according to the CIDI. European Psychiatry 1997;12:224-31.

79 Fan X, Wei J, Zhang J. On reliability and validity of social connectedness Scale-Revised in Chinese middle school students. Journal of Southwest China Normal University 2015;40:118-22.

80 Lee RM, Robbins SB. Measuring belongingness: the social connectedness and the social assurance scales. J Couns Psychol 1995;42:232-41.

81 Koenig HG, Westlund RE, George LK, et al. Abbreviating the Duke social support index for use in chronically ill elderly individuals. Psychosomatics 1993;34:61-9.

82 Zhou M, Qiu J, Mou Y. Reliability and validity of the 23-Duke social support scale among rural elderly population. Chinese Journal of Public Health 2015;31:1369-72. 
83 Hou F, Cerulli C, Wittink MN, et al. Depression, social support and associated factors among women living in rural China: a crosssectional study. BMC Womens Health 2015;15:28.

84 Zhang J, Conwell Y, Wieczorek WF, et al. Studying Chinese suicide with proxy-based data: reliability and validity of the methodology and instruments in China. J Nerv Ment Dis 2003;191:450.

85 Jia C, Zhang J. Psychometric characteristics of the Duke social support index in a young rural Chinese population. Death Stud 2012;36:858-69.

86 Link BG, Struening EL, Neese-todd S, et al. On describing and seeking to change the experience of stigma. Psychiatric Rehabilitation Skills 2002;6:201-31.

$87 \mathrm{Xu} \mathrm{H}$. A study of stigma of people with schizophrenia and relationship with medication compliance. Peking Union Medical College, 2008.

88 Zhang JX, Schwarzer R. Measuring optimistic self-beliefs: a Chinese adaptation of the general self-efficacy scale. Psychologia: An International Journal of Psychology in the Orient 1995;38:174-81.

89 Liamputtong P. Researching the vulnerable: a guide to sensitive research methods. Sage, 2006.
90 Liamputtong P. Qualittive research methods. Fourth ed. Australia: Oxford University Press, 2013: 218-37.

91 Inagaki M, Kawashima Y, Kawanishi C, et al. Interventions to prevent repeat suicidal behavior in patients admitted to an emergency department for a suicide attempt: a meta-analysis. J Affect Disord 2015;175:66-78.

92 Mann JJ, Apter A, Bertolote J, et al. Suicide prevention strategies: a systematic review. JAMA 2005;294:2064-74.

93 World Health Organization. Global health Observatory data Repository 2019. Available: http://apps.who.int/gho/data/?theme= main [Accessed 23 Oct 2019].

94 Zhao W, Zhu Y, Luo XW. The assertive community treatment model for severe mental disorders: a review. Chinese Mental Health Journal 2014;28:89-96

95 Shenzhen Mental Health Center. Shenzhen mental health work information brief Shenzhen: Shenzhen mental health center, 2020.

96 Schechter M, Goldblatt MJ, Ronningstam E, et al. Postdischarge suicide: a psychodynamic understanding of subjective experience and its importance in suicide prevention. Bull Menninger Clin 2016;80:80-96. 